\title{
ON A SPECIAL CLASS OF MEDIAN ALGEBRAS
}

\author{
MIGUEL COUCEIRO AND GERASIMOS C. MELETIOU
}

Received 09 October, 2015

\begin{abstract}
In this short note we consider a class of median algebras, called $(1,2: 3)$-semilattices, that is pertaining to cluster analysis. Such median algebras arise from a natural generalization of conservativeness, and their description is given in terms of forbidden substructures.
\end{abstract}

2010 Mathematics Subject Classification: 03C05; 06D75

Keywords: median algebra, median semilattice, generalized conservativeness

\section{PRELIMinaries: MEDian ALGEBras}

Recall that a median algebra is a structure $\mathbf{A}=\langle A, \mathbf{m}\rangle$ for a nonempty set $A$ and a ternary symmetric operation $\mathbf{m}: A^{3} \rightarrow A$, called median, that is a near-unanimity (i.e., $\mathbf{m}(x, x, y)=x$ ) and such that for every $x, y, z, t, u \in A$,

$$
\mathbf{m}(\mathbf{m}(x, y, z), t, u)=\mathbf{m}(x, \mathbf{m}(y, t, u), \mathbf{m}(z, t, u)) .
$$

Alternative axiomatizations were also proposed $[2,7,8]$. In particular, it can be shown that the following equation

$$
\mathbf{m}(\mathbf{m}(x, y, z), y, z)=\mathbf{m}(x, y, z)
$$

holds in every median algebra.

Such algebras have been studied by several authors and they were shown to be tightly related to several ordered structures such as semilattices and distributive lattices. Indeed, Sholander [9] showed that each element $a$ of a median algebra $\mathbf{A}$ gives rise to a median semilattice $\left\langle A, \leq_{a}\right\rangle$ where $\leq_{a}$ defined by

$$
x \leq_{a} y \quad \Longleftrightarrow \quad \mathbf{m}(a, x, y)=x .
$$

In such a semilattice, $a$ is the bottom element $\mathbf{A}_{a}:=\left\langle A, \leq_{a}\right\rangle$ and the induced semilattice operation $\wedge_{a}$ is then given by $x \wedge_{a} y=\mathbf{m}(a, x, y)$. Furthermore, its principal ideals

$$
\downarrow x:=\{y \in A: y \leq a x\}
$$

are distributive lattices. Conversely, if a $\wedge$-semilattice has the property that for any $a, b, c \in A$, the three elements $a \wedge b, b \wedge c, c \wedge a$ have a supremum whenever each pair 
of them is bounded above, then a median operation can be defined by

$$
\mathbf{m}_{\leq}(x, y, z)=(x \wedge y) \vee(x \wedge z) \vee(z \wedge y), \quad \text { for every } x, y, z \in A .
$$

Here $\vee$ denotes the supremum so that $\mathbf{m}_{\leq}(x, y, z)$ is defined as the supremum of $x \wedge y, x \wedge z$ and $z \wedge y$. As shown in [1], for every median algebra $\mathbf{A}=\langle A, \mathbf{m}\rangle$ and every $a \in A$, we have that $\mathbf{m}=\mathbf{m}_{\leq a}$.

Similarly, every distributive lattice gives rise to a median algebra using (1.1), and the converse also holds whenever there are $a, b \in A$ such that $\mathbf{m}(a, x, b)=x$ for every $x \in A$.

In the case of discrete structures, median algeras can also be thought of as certain special graphs. A median graph is a connected graph (i.e., for every pair of vertices there is a path connecting them) that has the property that for any three vertices $a, b, c$, there is exactly one vertex $x$ that minimizes the sum of the distances to $a, b$ and $c$. It was shown in [1] that every median semilattice whose intervals are finite has a covering graph (i.e., undirected HASSE diagram) that is median, and that every median graph is the covering graph of a median semilattice. For further background see, e.g., [2].

In this short note we are interested in some special classes of median algebras that are pertaining to cluster analysis, e.g., in the case of overlapping clusters. We say that a median semilattice $\mathbf{A}:=\langle A, \leq\rangle$ (thought of as a median algebra $\mathbf{A}=\langle A, \mathbf{m}\rangle$ ) is

(i) a (1:3)-median semilattice if for every $x, y, z \in A$,

$$
\mathbf{m}(x, y, z) \in\{x, y, z\}
$$

(ii) a (2:3)-median semilattice if for every $x, y, z \in A$,

$$
\mathbf{m}(x, y, z) \in\{x \wedge y, y \wedge z, z \wedge x\} ;
$$

(i i i ) a (1,2:3)-median semilattice if for every $x, y, z \in A$,

$$
\mathbf{m}(x, y, z) \in\{x, y, z, x \wedge y, y \wedge z, z \wedge x\} .
$$

Note that, apart from the 4-element Boolean lattice, every $(1: 3)$-median semilattice is a $(2: 3)$-median semilattice. Also, every $(1: 3)$-median semilattice and every $(2: 3)$-median semilattice is a $(1,2: 3)$-median semilattice.

However, the converse statements are not true as it is illustrated by Figure 1.

Remark 1 . Note that $(1: 3)$-median semilattices are exactly those median algebras whose subsets are themselves median (sub)algebras. In the terminology of [3], they are median algebras with $m s d$ (median stabilization degree) equal to 0 .

The median semilattices in $(i)$ and $(i i)$ were described in terms of forbidden substructures in [5] and [4], respectively. In this paper we provide a similar description for $(1,2: 3)$-median semilattices. 


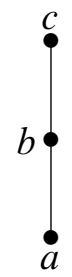

(a) $\mathbf{A}$

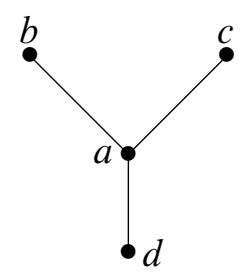

(b) $\mathbf{A}^{\prime}$

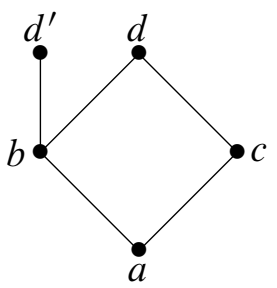

(c) $\mathbf{A}^{\prime \prime}$

FIgURE 1. Examples of median semilattices: $\mathbf{A}$ is a $(1: 3)$-median semilattice, $\mathbf{A}^{\prime}$ is a $(2: 3)$-median semilattice that is not $(1: 3)$, and $\mathbf{A}^{\prime \prime}$ is a $(1,2: 3)$-median semilattice that is not $(2: 3)$.

\section{MAIN RESUlT}

In [5], (1:3)-median semilattice were referred to as conservative median algebras, and it was shown that, apart from the 4-element Boolean algebra, they can be thought of as chains. (Curiously, the 4-element Boolean algebra also appears as an exceptional in another area of the theory of ordered sets; see e.g. [6].) Equivalently, (1:3)-median semilattices were shown to coincide exactly with those ordered structures that do not contain the the 4-element star $\mathbf{A}^{\prime}$ (see Figure 1(b)).

Recently, (2:3)-median semilattices were explicitly described in [4]. It was shown that $(2: 3)$-median semilattices coincide with those median semilattices that are trees: a $\wedge$-semilattice (resp. $\vee$-semilattice) is said to be a tree if no pair of incomparable elements have an upper (resp. lower) bound.

In particular, it follows that if a median algebra is a $(1: 3)$ - or a (2:3)-semilattice, then the same holds for any associated semilattice order $\leq a$. As illustrated by the ordered structures in Figure 2, this invariance no longer holds for $(1,2: 3)$-median semilattices.

As mentioned above, $(1: 3)$-median semilattices and (2:3)-median semilattices can be thought of as ordered structures that do not contain the 4-element star and the 4-element Boolean algebra, respectively. The following proposition provides a similar description for $(1,2: 3)$-median semilattices.

Proposition 1. Let $\mathbf{A}:=\langle A, \leq\rangle$ be a median semilattice. Then $\mathbf{A}$ is a $(1,2: 3)$ median semilattice if and only if it does not contain a copy of the median semilattice in Figure 2(b) as a semilattice (in fact, only need to consider b, $c, d$ and $d^{\prime}$ ).

Proof. Note that if $\mathbf{A}=\langle A, \leq\rangle$ contains a copy of the median semilattice in Figure 2(b), then taking $\{x, y, z\}=\left\{d^{\prime}, b, c\right\}$ have that

$$
\mathbf{m}(x, y, z) \notin\{x, y, z, x \wedge y, y \wedge z, z \wedge x\} .
$$

Hence the condition is necessary. 


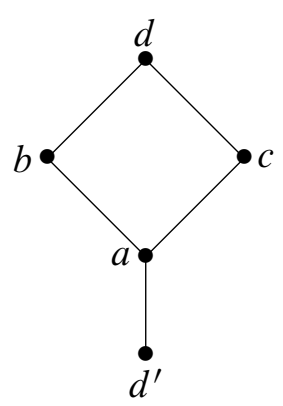

(a) $\mathbf{A}_{1}$

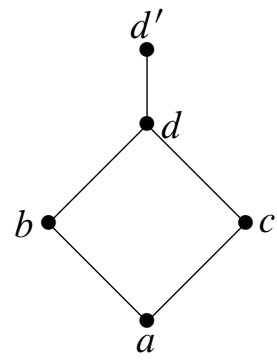

(b) $\mathbf{A}_{2}$

FIGURE 2. $\mathbf{A}_{1}$ is a $(1,2: 3)$-median semilattice, whereas the semilattice $\mathbf{A}_{2}$ is not.

To show that the condition is sufficient, suppose that $\mathbf{A}$ is not a (1,2:3)-median semilattice. Hence, there are $x, y, z, d \in A$ such that

$$
d=\mathbf{m}(x, y, z) \notin\{x, y, z, x \wedge y, y \wedge z, z \wedge x\} .
$$

Using the fact that

$$
d=(x \wedge y) \vee(y \wedge z) \vee(z \wedge x),
$$

we have that $b \wedge c<d$, for every $b, c \in\{x, y, z\}$ with $b \neq c$. Consider

$$
d^{\prime}=(x \wedge y) \vee(y \wedge z) \text {. }
$$

Clearly, $d^{\prime} \leq d$.

Now if $d^{\prime}<d$, then $\left\{x \wedge y, y \wedge z, x \wedge y \wedge z, d, d^{\prime}\right\}$ is a copy of Figure 2(b). So we may assume that $d=d^{\prime}=(x \wedge y) \vee(y \wedge z)=y \wedge(x \vee z)$. But in this case, $\{y, x \wedge y, y \wedge z, x \wedge y \wedge z, d\}$ is a copy of Figure 2(b). As these were the only two possible cases, the proof of Proposition 1 is now complete.

It is noteworthy that new examples of $(1,2: 3)$-median semilattices can be obtained from old ones by taking any non trivial bottom-rooted tree and replacing some of its leaves (maximal elements) by any (1,2:3)-semilattices, e.g., as in Figure 2(a). A further example can be obtained by taking, e.g., the $(1,2: 3)$-median semilattice in Figure 1(c) and replacing $d^{\prime}$ by the 4-element Boolean lattice.

\section{REFERENCES}

[1] S. P. Avann, "Metric ternary distributive semi-lattices," Proceedings of the American Mathematical Society, vol. 12, pp. 407-414, 1961, doi: 10.2307/2034206.

[2] H. J. Bandelt and J. Hedlíková, “Median algebras,” Discrete mathematics, vol. 45, pp. 1-30, 1983, doi: 10.1016/0012-365X(83)90173-5.

[3] H.-J. Bandelt and M. Van De Vel, “The median stabilization degree of a median algebra," Journal of Algebraic Combinatorics, vol. 9, no. 2, pp. 115-127, 1999, doi: 10.1023/A:1018689708341. 
[4] M. Couceiro, S. Foldes, and G. C. Meletiou, "Arrow type impossibility theorems over median algebras,", 2015, doi: arXiv:1508.04741v1.

[5] M. Couceiro, J.-L. Marichal, and B. Teheux, "Conservative median algebras and semilattices," Order, vol. 33, no. 1, pp. 121-132, 2016, doi: 10.1007/s11083-015-9356-x.

[6] S. Foldes and J. Szigeti, "A half-space approach to order dimension," Order, vol. 24, pp. 59-73, 2007, doi: 10.1007/s11083-007-9059-z.

[7] J. R. Isbell, "Median algebra," Transactions of the American Mathematical Society, vol. 260, no. 2, pp. 319-362, 1980, doi: 10.2307/1998007.

[8] M. Kolibiar and T. Marcisová, "On a question of J. Hashimoto," Mat. Časopis, vol. 24, pp. 179-185, 1974.

[9] M. Sholander, "Medians, lattices, and trees," Proc. American Mathematical Society, vol. 5, no. 5, pp. 808-812, 1954.

Authors' addresses

Miguel Couceiro

LORIA (CNRS - Inria Nancy Grand Est - Université de Lorraine), Équipe Orpailleur, Batiment B, Campus Scientifique, B.P. 239, F-54506 Vandoeuvre les Nancy

E-mail address: miguel.couceiro[at] inria.fr

Gerasimos C. Meletiou

TEI of Epirus, PO Box 110, 47100 Arta, Greece

E-mail address: gmelet [at] teiep.gr 